\title{
Notch 3 Protein, not its Gene Polymorphism, is Associated with the Chemotherapy Response and Prognosis of Advanced NSCLC Patients
}

\author{
Chunlei Shi Jialin Qian Meili Ma Yanwei Zhang Baohui Han \\ Department of Pulmonary Medicine, Shanghai Chest Hospital, Shanghai Jiaotong University, Shanghai, \\ China
}

\section{Key Words}

$\mathrm{NOTCH} 3 \cdot$ Non-small cell lung cancer $•$ Chemotherapy $•$ Prognosis

\begin{abstract}
Aim: To study the relation of NOTCH3 and its gene polymorphisms with the chemotherapy response and the prognosis of patients with Non-small cell lung cancer (NSCLC). Methods: A total of 594 patients with advanced stage of NSCLC (IIIA, IIIB and IV) NSCLC were enrolled. All patients received Platinum-based chemotherapy. The NOTCH3 expression in tumors and its gene polymorphisms were determined. In vitro, several NSCLC cell lines received the NOTCH3 over-expression vector and small interfering RNA (siRNA) to study the role of NOTCH3 in regulation the cellular biological behaviors. Results: The genotype and the allele frequencies of NOTCH3 gene polymorphisms at $605 \mathrm{C}>\mathrm{T}$ and $1735 \mathrm{~T}>\mathrm{C}$ were not significantly different between good responders and poor responders to chemotherapy. However, high NOTCH3 expression in tumor represented a significantly higher possibility of being resistant to chemotherapy. Also, patients with high NOTCH3 expression had a poorer prognosis than those with low NOTCH3 expression. In vitro studies showed that NOTCH3 inhibition dramatically suppressed the proliferation, migration, invasiveness abilities and prompted apoptosis in NSCLC cells. Conclusion: NOTCH3 regulates the cellular behavior, including, proliferation, migration, invasiveness abilities and apoptosis of NSCLC cells. NOTCH3 may be used as a marker to predict the chemotherapy response and prognosis of advanced NSCLC.
\end{abstract}


Shi et al:: The Notch 3 Protein and Chemotherapy Response in Advanced NSCLC

\section{Introduction}

Platinum-based chemotherapy is the standard first-line chemotherapy for advanced Non-small cell lung cancer (NSCLC). However, more than one third patients receiving Platinum-based chemotherapy encounter drug resistance, which dramatically influences the clinical outcome of patients [1-3]. The identification of new maker predicting the chemotherapy response is important to improve the prognosis of patients with NSCLC. Recent studies show that the individual genetic background of patients plays important role in determining the chemotherapy response in NSCLC patients [4-8].

NOTCH3 is a member of the NOTCH signaling pathway which includes NOTCH ligands, NOTCH receptors, and transcription factors [9]. NOTCH signaling activation has been demonstrated to be involved in carcinogenesis $[10,11]$. Aberrant activations of Notch signaling pathways are associated with the pathogenesis of NSCLC [12]. The NOTCH3 is overexpressed in NSCLC cell lines and this over-expression is accompanied by chromosome 19 translocation [13]. In contrast, inhibition of the Notch3 pathway dramatically reduces cancer cell growth via decreasing phosphorylation of the mitogen-activated protein kinase [14]. In a genetically engineered murine models of NSCLC, tumor cells with induced expression of NOTCH3 had a increased tumorigenicity [15]. A recent study shows that NOTCH3 overexpression is related to the recurrence of ovarian cancer and confers resistance to carboplatin [16], suggesting that NOTCH3 may be used as a prognostic biomarker and therapeutic target in ovarian cancer.

Two genetic polymorphisms of NOTHC3 gene have been reported. The $605 \mathrm{C}>\mathrm{T}$ polymorphism locates in exon 4 and causes an alanine to valine amino acid change at position 202. The $1735 \mathrm{~T}>\mathrm{C}$ mutation locates in exon 11 and causes a cysteine to arginine amino acid change at position 579 [17]. To date, little is known about the relation between tumor NOTCH3 expression and its gene polymorphisms and the chemotherapy response, clinical feature and prognosis of NSCLC. In this study, we enrolled advanced NSCLC patients undergoing Platinum-based chemotherapy to test this association. Additionally we also conducted in vitro experiment to explore the role of NOTCH3 in cell biological behavior of cancer cells.

\section{Materials and Methods}

\section{Patient enrollment}

A total of 594 patients with advanced stage of NSCLC, namely, stage III (A+B) and IV NSCLC, confirmed cytologically or histologically were enrolled into this study. To avoid the potential influence of poor clinical conditions on chemotherapy response, other eligibility criteria included: normal blood chemistries (hemoglobin $>9 \mathrm{~g} / \mathrm{dl}$, neutrophil count $>1500 / \mathrm{mm} 3$ and platelet count $>100000 / \mathrm{mm} 3$ ), hepatic function (bilirubin $<1.5$ times the normal upper limit, aspartate aminotransferase and alanine aminotransferase $<2$ times the normal upper limit) and renal function (Creatine clearance rate $>50 \mathrm{ml} / \mathrm{s}$ ), and normal electrocardiogram at the beginning of treatment. Smokers were defined as those who had smoked more than one cigarette per day and more than 1 year in their lifetime. Exclusion criteria included symptomatic brain metastases, spinal cord compression, uncontrolled massive pleural effusion and previously received chemotherapy and other chronic disease. The study was approved by the ethics committees of our hospital and written informed consent was obtained from each participant.

\section{Chemotherapy regimens and therapeutic effect evaluation}

All patients had received platinum-based chemotherapy after diagnosis. Patient responses to treatment were determined by the WHO criteria [18], which classify the response into four categories: complete response (CR), partial response (PR), stable disease (SD), and progressive disease [19]. CR and PR were combined as responders, and SD and PD were grouped as non-responders. The chemotherapy response was assessed by two independent oncologists who were blind to our study. Overall survival (OS) and progression free survival (PFS) were the end points in this study. OS was calculated from the date of 
Shi et al.: The Notch 3 Protein and Chemotherapy Response in Advanced NSCLC

chemotherapy to the date of last follow-up or death from any cause. PFS was defined as the interval between the date of chemotherapy and the date of confirmed relapse.

\section{NOTCH3 genotyping}

DNA was extracted from peripheral whole blood using a Qiagen DNA Isolation Kit (Qiagen, Valencia, CA, USA). The NOTCH3 sequencing was performed as previously described [20]. Briefly, $5 \mu \mathrm{l}$ of PCR product was made to a total of $10 \mu \mathrm{l}$ reaction. The cycling conditions for this step were: $\left(37^{\circ} \mathrm{C}-15 \mathrm{~min}\right)-1 \mathrm{cycle}$, $\left(80^{\circ} \mathrm{C}-15 \mathrm{~min}\right)-1 \mathrm{cycle}$ and $\left(4{ }^{\circ} \mathrm{C}-2 \mathrm{~min}\right)-1$ cycle. The subsequent step was the labelling reaction carried out using ABI BigDye ${ }^{\mathrm{TM}}$ Terminator (BDT) v3.1 Cycle Sequencing chemistry. Cycling protocol for BDT reaction: $\left(96^{\circ} \mathrm{C}-1 \mathrm{~min}\right)-1 \mathrm{cycle},\left(96^{\circ} \mathrm{C}-10 \mathrm{~s}, 50^{\circ} \mathrm{C}-5 \mathrm{~s}, 60^{\circ} \mathrm{C}-4 \mathrm{~min}\right)-30$ cycles, and $\left(4{ }^{\circ} \mathrm{C}-5 \mathrm{~min}\right.$, $10{ }^{\circ} \mathrm{C}-5 \mathrm{~min}$ and $4{ }^{\circ} \mathrm{C}-2 \mathrm{~min}$ ) -1 cycle. Following this, the samples were purified and concentrated using ethanol precipitation with sodium acetate, EDTA and ethanol. We performed sequencing on an ABI 3130 Genetic Analyser .

\section{Immunohistochemical analysis and evaluation}

Tumor samples were obtained from the biopsy before the start of chemotherapy. The non-cancerous lung tissues were obtained from 14 patients receiving biopsy due to non-tumor diseases. The tissues were fixed, paraffin embedded, and cut to 5- $\mu \mathrm{m}$-thick sections for immunohistochemistry. Briefly, the slides were incubated with Rabbit polyclonal antibody to NOTCH3 (1:200, Abcam (ab60087) USA). The immunoreactive products were visualized by the catalysis of 3,3'-diaminobenzidine . NOTCH3 expression were classified semiquantitatively by immunoreactive score according to the method described previously [21]. NOTCH3 staining was defined as low expression (IRS: 0-5) and high expression (IRS: 6-12).

Tumor Cell line Culture and small interfering RNA (siRNA) transfection for NOTCH3

Three human NSCLC cell lines, namely, H292, A549, Calu-3 were cultured to $90 \%$ confluence before small interfering RNA (siRNA) transfection. The double-stranded short interfering RNA (siRNA) for NOTCH3 (50-GUCAAUGUUCACUUCGCAGUU-30 and 50-GCGUGGAUUCGGACCAGUCUGAGAGGG-30) was synthesized from Genechem (Shanghai. China).

NOTCH3 over-expression retrovirus vector

The NOTCH3 intracellular domain (NICD3; amino acids 1663 to 2312) retrovirus vector was a gift from Dr Xiaohau Huang from Dalian medical university. Briefly, the PCR product of the NOTCH3 intracellular domain was cloned into an expression vector, pcDNA6, with a V5 tag. The coding sequence of NICD3-V5 was subcloned into the pBabe-puro retroviral vector, and high-titer retroviral stocks were generated [22]. Cells infected with the empty pBabe-puro retroviral vector were used as controls.

\section{MTT Cell Proliferation Assay}

Cells were seeded in a 96-well plate right after si-RNA transfection. The medium was discarded and $100 \mu \mathrm{l}$ of dimethyl sulfoxide was added to each well. Absorbance was measured at a wavelength of $562 \mathrm{~nm}$ using an ELISA microplate reader (Bio-Rad, Hercules, CA, USA). Assays were repeated at least three times.

\section{Cell invasion and migration assay}

The cell invasion and migration ability was evaluated using transwell inserts with $8 \mu \mathrm{m}$ pores (BD Biosciences, San Jose, CA, USA). For invasion assay, $2 \times 10^{5}$ cells in serum free medium were added to each upper compartment of the chamber pre-coated with matrigel matrix (BD Biosciences, San Jose, CA, USA). After incubation for 48 hours, noninvasive cells were removed from the upper surface of the transwell membrane, and migrated cells were fixed with methanol, stained with Giemsa and photographed under the microscope. For migration assay, $2 \times 10^{5}$ cells were placed into the top chamber without matrigel matrix pre-coated. Finally, the cells in lower compartment of the chamber that had invaded to the basal side of the membrane were counted using a light microscope in 5 random visual fields $(\times 200)$.

Terminal Deoxynucleotidyl Transferase dUTP Nick End Labeling (TUNEL) Assay

The apoptotic cells in heart tissue and cultured cells were detected by in situ DeadEndTM Colorimetric Apoptosis Detection System (Promega, Madison, WI) according to the manufacturer's instructions. Briefly, 
Shi et al.: The Notch 3 Protein and Chemotherapy Response in Advanced NSCLC

Table 1. NSCLC Patient Characteristics. NS (not significance), DDP (cisplatin), CBP (carboplatin), TAX (taxol/paclitaxel), TXT (taxetere), DOC (docetaxel), GEM (gemcitabine), NVB (vinorelbine)

\begin{tabular}{|c|c|c|c|c|c|}
\hline Characteristics Patient & Responder & & Non-responder & & Pvalue \\
\hline & $n=214$ & & $n=380$ & & \\
\hline Age (years) & $54.6 \pm 2.7$ & & $54.7 \pm 2.2$ & & NS \\
\hline$B M I$ & $24.1 \pm 3.6$ & & $23.8 \pm 4.2$ & & NS \\
\hline \multicolumn{6}{|l|}{ Gender } \\
\hline Male & 134 & $62.62 \%$ & 250 & $65.79 \%$ & \multirow[t]{2}{*}{0.246} \\
\hline Female & 80 & $37.38 \%$ & 130 & $34.21 \%$ & \\
\hline \multicolumn{6}{|l|}{ Smoke status } \\
\hline Never smokers & 160 & $74.77 \%$ & 250 & $64.43 \%$ & \multirow[t]{2}{*}{$<0.001$} \\
\hline Ever Smoker & 54 & $25.23 \%$ & 138 & $35.57 \%$ & \\
\hline \multicolumn{6}{|l|}{ Histology } \\
\hline Squamous cell carcinoma & 130 & $60.75 \%$ & 216 & $56.84 \%$ & \multirow[t]{2}{*}{0.201} \\
\hline Adenocarcinoma & 84 & $39.25 \%$ & 164 & $43.16 \%$ & \\
\hline \multicolumn{6}{|l|}{ Stage } \\
\hline IIIA & 87 & $40.65 \%$ & 90 & $23.68 \%$ & \multirow[t]{3}{*}{$<0.001$} \\
\hline IIIB & 93 & $43.46 \%$ & 178 & $46.84 \%$ & \\
\hline IV & 34 & $15.89 \%$ & 112 & $29.47 \%$ & \\
\hline \multicolumn{6}{|l|}{ Differentiation } \\
\hline Well & 79 & $36.92 \%$ & 103 & $27.11 \%$ & \multirow[t]{3}{*}{$<0.001$} \\
\hline Moderate & 107 & $50.00 \%$ & 180 & $47.37 \%$ & \\
\hline Poor & 28 & $13.08 \%$ & 97 & $25.53 \%$ & \\
\hline \multicolumn{6}{|l|}{ Chemotherapy regimens } \\
\hline$D D P / C B P+T A X / T X T / D O C$ & 78 & $36.45 \%$ & 118 & $31.05 \%$ & \multirow[t]{3}{*}{0.114} \\
\hline$D D P / C B P+G E M$ & 102 & $47.66 \%$ & 176 & $46.32 \%$ & \\
\hline$D D P / C B P+N V B$ & 34 & $15.89 \%$ & 86 & $22.63 \%$ & \\
\hline
\end{tabular}

sample slides were washed in PBS and then fixed in $4 \%$ paraformaldehyde solution. Sections were incubated with terminal deoxynucleotidyl transferase enzyme in a humidified chamber at $37{ }^{\circ} \mathrm{C}$ for $60 \mathrm{~min}$. The reaction was terminated by transferring the slides to $2 \times$ sodium citrate saline solution. The sections were counter stained with DAPI. For quantitative analyses, 5 fields per section were selected. Apoptosis was indexed by counting TUNEL positive cells per 100 nuclei per section [23].

\section{Cisplatin cytotoxicity assay}

Cell lines were seeded at $1 \times 10^{4}$ cells per well in 96-well plates, and were transfected by NOTCH3 siRNA and NOTCH3 over-expression retrovirus vector for $48 \mathrm{~h}$, and subsequently exposed to cisplatin at final concentrations of $0.5,1.0,2.0,4.0$ or $8.0 \mathrm{ug} / \mathrm{ml}$ for $24 \mathrm{~h}$ in triplicate wells. Cell survival was determined using a previously described colorimetric MTT assay. Mean cell viability was calculated by the ratio of absorbance units of transfected cell samples to the mean absorbance units of the control cell samples. All the experiments were repeated at least three times. The IC50 value is defined as concentration of cisplatin that is required for a $50 \%$ reduction in absorbance calculated from the growth curves.

\section{Statistical Analyses}

Association between NOTCH3 expression status and clinical parameters were studied using chisquare test, or independent $t$ tests. Multivariate analysis was performed using the Cox proportional hazards model selected in forward stepwise. The hazard ratio (HR) (OR) and 95\% confidence intervals (CIs) were calculated. Survival was analyzed using the Kaplan-Meier method. The log-rank test was used to analyze survival differences. The data in proliferation rate, migration, invasiveness, cisplatin cytotoxicity assays between NOTCH3 si-RNA, NOTCH3 over-expression vector and their controls were compared by using $t$ tests. $\mathrm{P}<0.05$ was considered statistically significant. All analyses were performed by using SAS software (version 9.2, USA).

\section{Results}

\section{NSCLC Patient Characteristics}

Among all enrolled patients, 214 patients were assigned as good responders (CR + PR) and 380 were poor responders to chemotherapy (SD + PD). No significant differences were noted in mean age, gender distribution, histology type and chemotherapy agent between the chemotherapy responder and non-responders (Table 1). However, the poor responders 
Table 2. Genotype frequencies of NOTCH3 polymorphisms and chemotherapy response in NSCLC patients

\begin{tabular}{rrccccc}
\hline & \multicolumn{3}{c}{ Responder } & \multicolumn{4}{c}{ Non-responder } & P value \\
& & N=214 & $\%$ & N=380 & $\%$ & \\
\hline $605 C>$ T & CC & 59 & $26.94 \%$ & 113 & $29.74 \%$ & 0.573 \\
& CT & 110 & $50.23 \%$ & 174 & $45.79 \%$ & \\
& TT & 50 & $22.83 \%$ & 93 & $24.47 \%$ & \\
& C & 228 & $52.05 \%$ & 400 & $52.63 \%$ & 0.447 \\
& T & 210 & $47.95 \%$ & 360 & $47.37 \%$ & \\
$1735 \mathrm{~T}>\mathrm{C}$ & TT & 55 & $25.11 \%$ & 110 & $28.95 \%$ & 0.397 \\
& TC & 108 & $49.32 \%$ & 189 & $49.74 \%$ & \\
& CC & 56 & $25.57 \%$ & 81 & $21.32 \%$ & \\
& T & 218 & $49.77 \%$ & 409 & $53.82 \%$ & 0.099 \\
& C & 220 & $50.23 \%$ & 351 & $46.18 \%$ & \\
\hline
\end{tabular}

Table 3. NOTCH3 expression status in NSCLC patients

\begin{tabular}{lccc}
\hline & $\begin{array}{c}\text { High } \\
\text { NOTCH3 } \\
\text { expression }\end{array}$ & $\begin{array}{c}\text { Low NOTCH3 } \\
\text { expression }\end{array}$ & $\begin{array}{c}\text { Adjusted } \\
\text { HR }\end{array}$ \\
\hline Stage & 44 & 34 & 1 \\
IIIA & 43 & 50 & 0.784 \\
IIIB & 24 & 10 & 2.012 \\
IV & & 30 & 1 \\
Differentiation & 49 & 46 & 0.872 \\
Moderate & 61 & 4 & 3.543 \\
Poor & 24 & & \\
Chemotherapy & & & \\
response & & 147 & 1 \\
responder & 67 & 69 & 8.765 \\
Non-responder & 311 & & \\
\hline
\end{tabular}

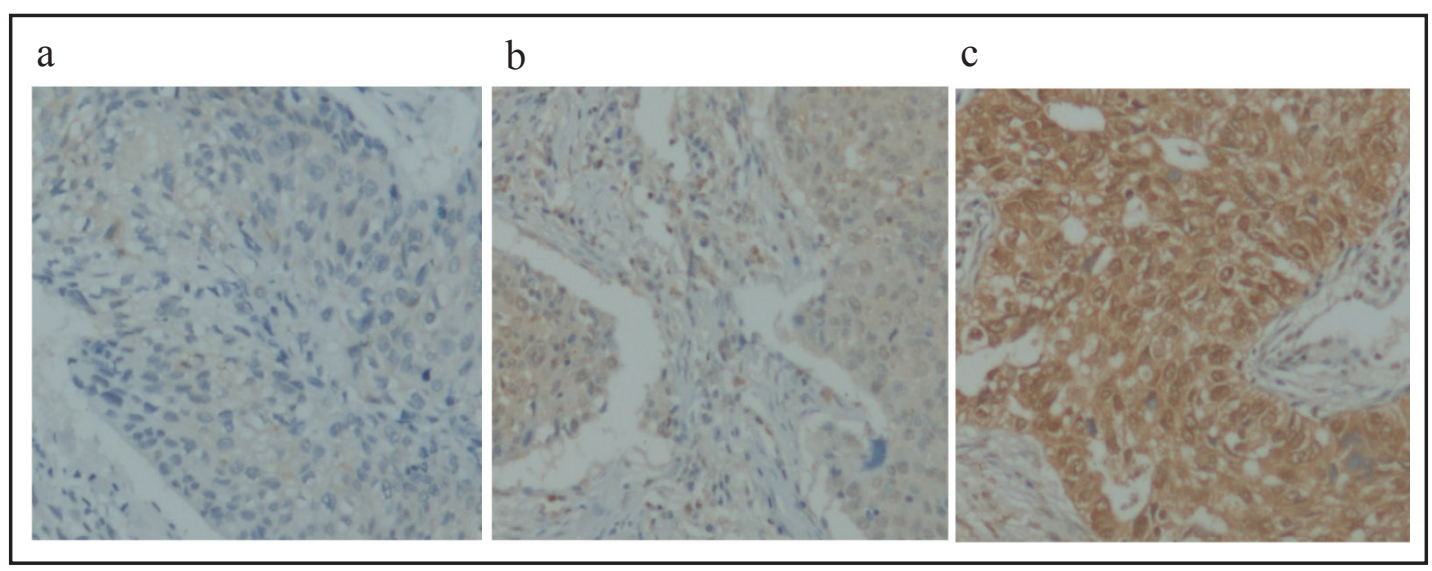

Fig. 1. Typical NOTCH3 expression in tumor sample. (a) Weak NOTCH3 expression in non-cancerous lung tissue sample. (b) Low NOTCH3 expression in NSCLC tumor sample. (c) High NOTCH3 expression in NSCLC tumor sample.

had higher percentage for ever smokers, higher tumor stage, poorer differentiation than responders (all $\mathrm{P}<0.001$ ).

Genotype frequencies of NOTCH3 polymorphisms in good responder andpoor responders were found to be in Hardy-Weinberg equilibrium (all $\mathrm{P}>0.05$ ). The genotype and the allele frequencies of $605 \mathrm{C}>\mathrm{T}$ and $1735 \mathrm{~T}>\mathrm{C}$ were not significantly different between two groups (all $\mathrm{P}>0.05$, Table 2). In additional the NOTCH3 gene polymorphism did not correlate to the clinical features either (all $\mathrm{P}>0.05$, data not shown).

Typical NOTCH3 staining in tumor samples is shown in Fig. 1. NOTCH3 is mainly localized in the cytoplasm and cell membrane. NOTCH3 is weakly expressed in the non-cancerous lung tissues. Of 214 good responders, 67 (31.31\%) had high NOTCH3 expression. In contrast, of the poor responders, there were 311 (81.84\%) had high NOTCH3 expression. Multivariate logistic regression analysis reveals that high NOTCH3 expression represented a significantly higher possibility to be non-responder to chemotherapy in NSCLC patients with adjustment with age, sex, smoking status, histology, differentiation, chemotherapy regimens (adjusted $\mathrm{OR}=8.765$, 95CI: 6.654-11.543, adjusted $\mathrm{P}<0.001$ ). Also, NOTCH3 is associated with higher tumor stage (Adjusted $\mathrm{OR}=2.012, \mathrm{P}=0.0013$ ), and poorer differentiation status (adjusted $\mathrm{OR}=3.543, \mathrm{P}<0.001)$.

Prognosis analyses shows that patients with high NOTCH3 expressions had markedly shorter PFS and OS than those with low NOTCH3 expressions (PFS: $7.9 \pm 3.6$ vs. $9.8 \pm 4.1$, months, and OS: $10.4 \pm 3.8$ vs. $16.7 \pm 3.1$, months, both $\mathrm{P}<0.001$ by log-rank test). KaplanMeier survival curves for PFS and OS are shown in Fig. 2a and 2b, respectively. 
$\mathrm{a}$

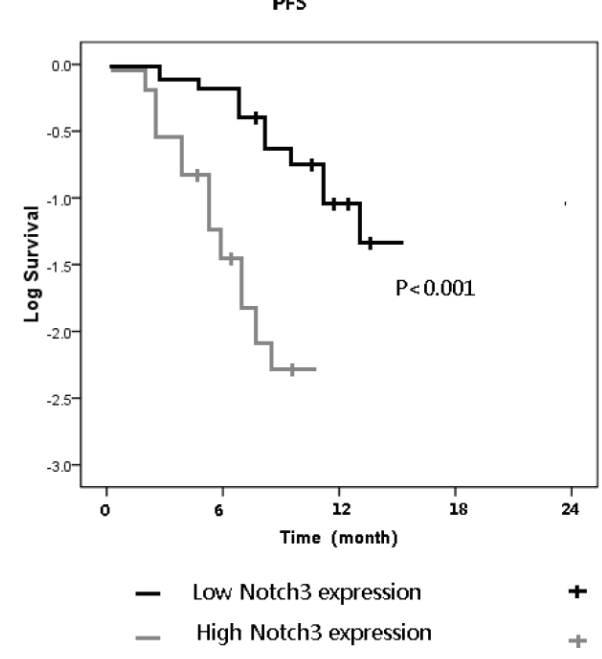

b

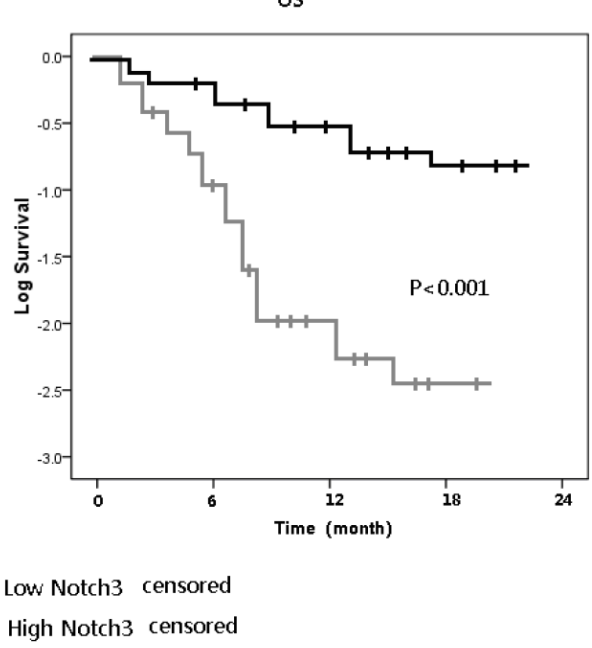

Fig. 2. Kaplan-Meier survival curves for PFS and OS in NSCLC patients. (a) shows the survival curves for PFS. Patients with high NOTCH3 expressions $(n=111)$ had markedly shorter PFS than those with low NOTCH3 expressions ( $\mathrm{n}=94,7.9 \pm 3.6$ vs. $9.8 \pm 4.1$, months, $\mathrm{P}<0.001$ ). (b) shows the survival curves for OS. Patients with high NOTCH3 expressions had markedly shorter OS than those with low NOTCH3 expressions $(10.4 \pm 3.8$ vs. 16.7 \pm 3.1 , months, $\mathrm{P}<0.001$ ).

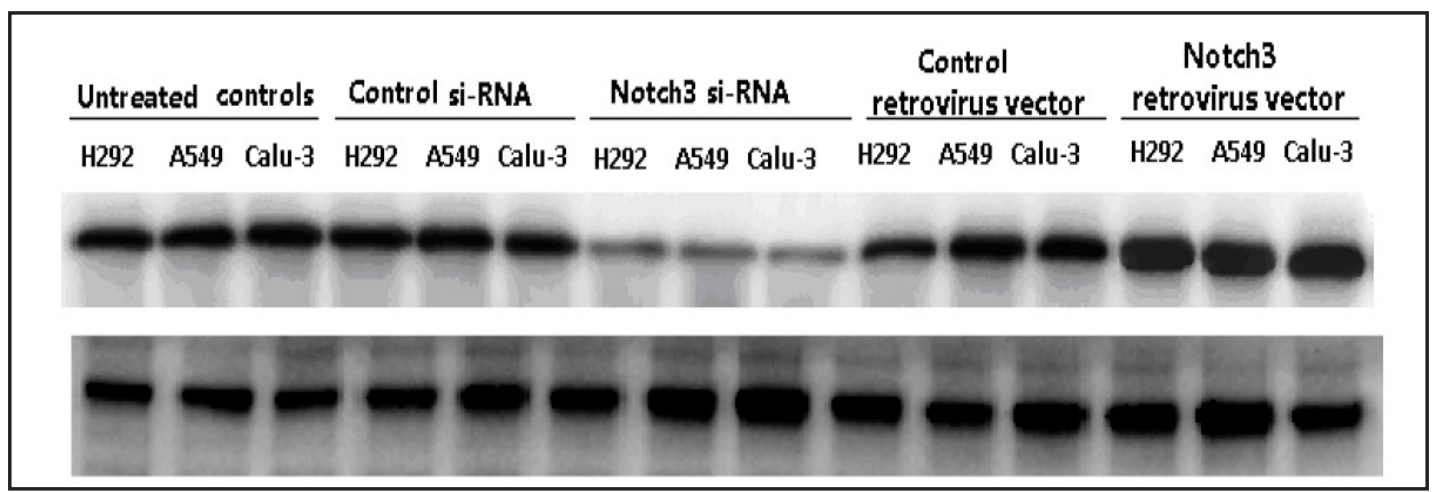

Fig. 3. The NOTCH3 protein expressions in NSCLC cell lines treated by si-RNA and over-expression vector. Fig. 3 shows the Western blot results of NOTCH3 protein expressions in NSCLC cell lines. We found that NOTCH3 si-RNA silencing technique significantly inhibited the NOTCH3 expression, while the NOTCH3 retrovirus vector transfection dramatically increased NOTCH3 expressions in H292,A549 and Calu-3 cell lines.

Univariate and multivariate analyses were performed by using Cox proportional hazard model to evaluate the impact of NOTCH3 expression on the prognosis of NSCLC (Table 4). Univariate and multivariate analyses confirmed that NOTCH3 expression was associated with the PFS and OS of NSCLC patients (Table 3). Patients with low NOTCH3 expression had poorer prognosis (PFS: adjusted HR=2.675, adjusted $\mathrm{P}<0.001$; OS: adjusted HR=4.889, adjusted $\mathrm{P}<0.001)$. In addition, the tumor stage and differentiation status is responsible for the PFS and OS as well (Table 4).

NOTCH3 inhibition and over-expression on the proliferation, migration and invasion abilities as well as apoptosis of NSCLC cell lines

Western blot results show that the NOTCH3 protein expressions in NSCLC cell lines were significantly inhibited by si-RNA silencing technique (Fig. 3) and dramatically increased by NOTCH3 retrovirus vector transfection. The cell proliferation assays revealed the cell growth 
Table 4. Multivariate Cox proportional regression analysis on PFS and OS of NSCLC patients

\begin{tabular}{lcccccc}
\hline Variable & PFS & & \multicolumn{2}{c}{ OS } & \\
& HR & 95\%CI & P & HR & $95 \%$ CI & P value \\
\hline $\begin{array}{l}\text { NOTCH3 expression } \\
\text { Low expression }\end{array}$ & 1 & & & 1 & & \\
High expression & 2.675 & $2.332-4.883$ & $<0.001$ & 4.889 & $2.248-8.376$ & $<0.001$ \\
Tumor stage & 1 & & & 1 & & \\
IIIA+IIIB & 2.357 & $2.227-4.427$ & 0.0011 & 2.587 & $1.262-3.543$ & 0.008 \\
IV & & & & & & \\
Tumor differentiation & 1 & & & 1 & & \\
Well+Moderate & 3.132 & $2.143-4.876$ & $<0.001$ & 2.262 & $1.624-3.264$ & $<0.001$ \\
Poor & & & & & & \\
\hline
\end{tabular}

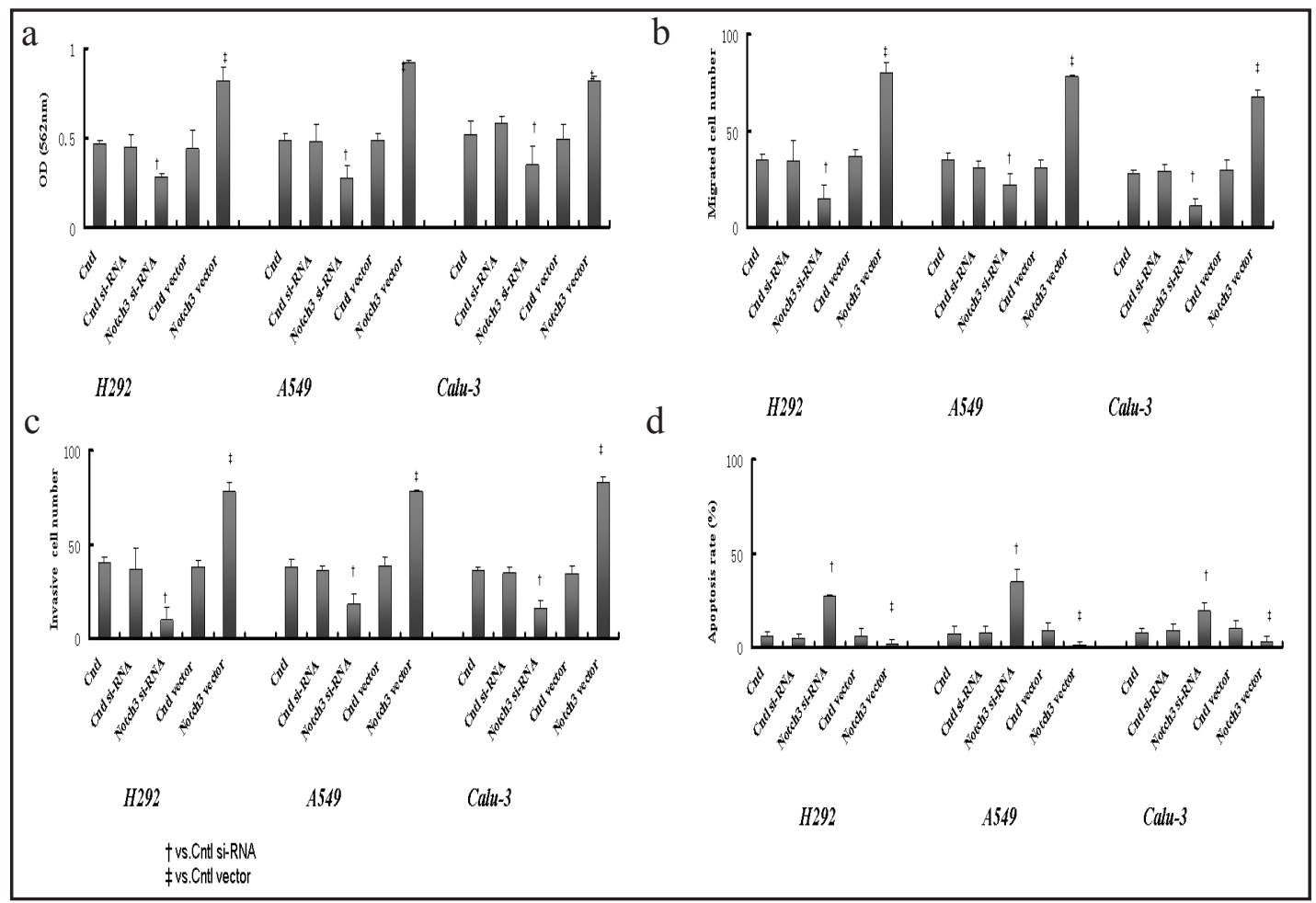

Fig. 4. NOTCH3 inhibition and over-expression on the proliferation, migration and invasion abilities as well as apoptosis of NSCLC cell lines. (a) shows the cell proliferation assays by MTT assay. Our data reveals that the cell growth rate were significantly decreased in all NSCLC cell lines receiving NOTCH3 si-RNA transfection, meanwhile, they are significantly increased after NOTCH3 over-expression. (b and c) shows the cell migration and invasiveness abilities in NSCLC cell lines. NOTCH3 si-RNA transfection markedly decreased the cell migration and invasiveness abilities in all NSCLC cell lines. In contrast, NOTCH3 over-expression dramatically increased cell migration and invasiveness abilities in these cells. (d) shows the cell apoptosis in NSCLC cells. NOTCH3 si-RNA transfection markedly increased the numbers of TUNUL positive cells in studied NSCLC cell lines, which are markedly decreased by NOTCH3 over-expression

rate, migration and invasiveness abilities were all significantly decreased in NSCLC cell lines receiving NOTCH3 si-RNA transfection. Meanwhile, they are significantly increased after NOTCH3 over-expression (Fig. 4a-c). However, the apoptosis rate was dramatically increased by NOTCH3 inhibition and decreased by NOTCH3 over-expression in these cells (Fig. 4d).

The chemosensitivity of NSCLC cell lines to cisplatin after Notch3 inhibition and overexpression

Exposure of H292, A549, Calu-3 cells receiving NOTCH3 si-RNA transfection and overexpression cells to different concentrations of cisplatin induced significant proliferative 
Fig. 5. The chemosensitivity of NSCLC cell lines to cisplatin after NOTCH3 inhibition and over-expression. (a-c) show the chemosensitivity of NSCLC cell lines to cisplatin after NOTCH3 inhibition and over-expression. Exposure of H292, A549, Calu-3 cells receiving NOTCH3 siRNA transfection and overexpression cells to different concentrations of cisplatin induced significant proliferative inhibition $(\mathrm{a}, \mathrm{b}$ and $\mathrm{c}$, respectively). The IC50 concentration of cisplatin for NOTCH3 si-RNA transfected cells decreased significantly than control si-RNA cells (all $\mathrm{P}<0.001$ ), and that for NOTCH3 over-expression markedly increased the IC50 concentration of cisplatin in these cell lines compared to control vector transfection (all $\mathrm{P}<0.001$ ).

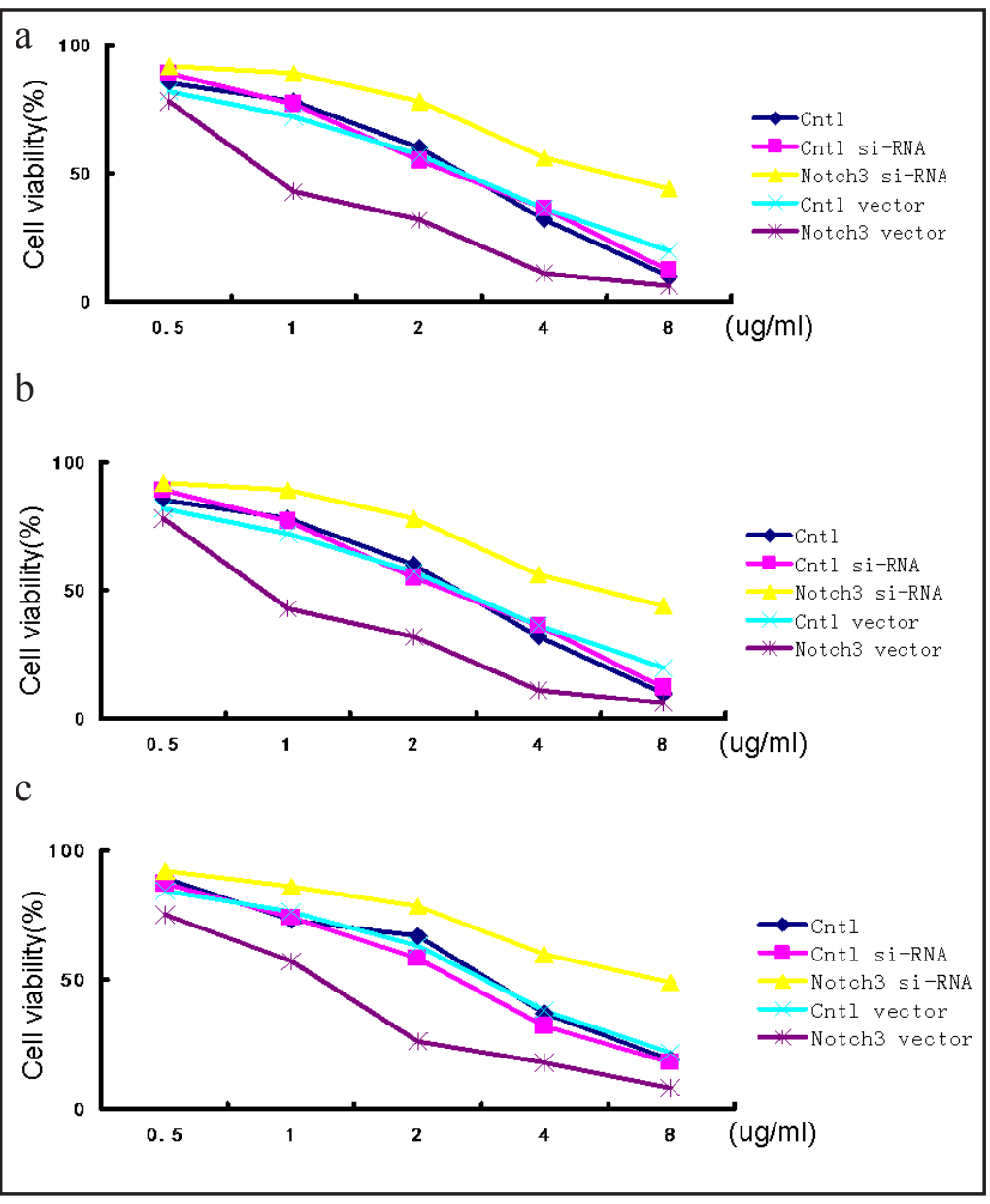

inhibition (Fig. 5 a,b and c, respectively). The IC50 concentration of cisplatin for NOTCH3 si-RNA transfected cells decreased significantly than control si-RNA cells (all $\mathrm{P}<0.001$ ), and that for NOTCH3 over-expression markedly increased the IC50 concentration of cisplatin in these cell lines compared to control vector transfection (all $\mathrm{P}<0.001$ ).

\section{Discussion}

Notch signaling pathway plays an important role in the normal lung development and the dysregulation of Notch signaling has been implicated in various lung diseases including lung cancer [24]. Previous studies have shown that the over-expression of NOTCH3 is associated with poor prognosis in human NSCLC patients $[25,26]$. However, it is not clear whether the expression level of NOTCH3 in tumor is related to the treatment response to Platinum based chemotherapy. Our results show that high NOTCH3 expression represented significantly higher possibility for being poor responder to chemotherapy in NSCLC patients with adjustment with age, sex, smoking status, histology, differentiation. Moreover, higher tumor NOTCH3 expression predicted poor PFS and OS in these patients.

Our in vitro studies show that the over-expression of NOTCH3 is related to increased cell growth rate, migration and invasiveness abilities, but decreased apoptosis rate in three NSCLC cell lines. Meanwhile, we also found that NOTCH3 si-RNA transfection in these NSCLC cell lines reversed these above mentioned cellular biological behaviors. In addition, the chemosensitivity of these NSCLC cells changed with their NOTCH3 expression levels. These in vitro data, combined with our clinical data, suggest that activation of the NOTCH3 is involved in the regulation of NSCLC cancer cell behaviors and the chemosensitivity to anti- 
tumor agents, thus determine the chemotherapy response in NSCLC patients.

It is reported that the polymorphism in the NOTCH3 gene is related with cerebral autosomal dominant arteriopathy with subcortical infarcts and leukoencephalopathy [27]. However, whether the polymorphism of NOTCH3 is related with chemotherapy response in NSCLC patients remain unknown. Our results did not show positive association of the polymorphism of NOTCH3 gene with the clinical feature and chemotherapy response in NSCLC patients. There results confirmed that it is the expression level of Notch 3 protein, not its gene polymorphism, which is associated with the chemotherapy response of advanced NSCLC patients.

Previous studies showed that pharmacologic treatment of mice carrying autochthonous NSCLCs with a $\gamma$-secretase inhibitor (GSI), a notch signal inhibitor, blocks cancer growth [28] [29]. Our results show that the chemosensitivity of NSCLC cells increase when the expression level of NOTCH3 was down-regulated. Therefore, our results suggest that NOTCH3 signal could be a therapeutic target for the chemotherapy non-responders in NSCLC patients.

Several limitations should be addressed in this study. Firstly, this is a single center study and the sample size is relatively small. Thus, the utility of NOTCH3 expression as markers for chemotherapy response and prognosis of NSCLC patients warrants further confirmation.

Secondly, like the other Notchs, the nuclear localization of NOTCH3 is essential for its activation. In this study, we detected only total expression of NOTCH3. The nuclear translocation of NOTCH3 from cytoplasma into nucleis was not analyses. This is another major limitation of this study.

In summary, our data show that NOTCH3 participates in regulation of chemosensitivity to cisplatin in NSCLC cells. Over-expression of NOTCH3 in tumor is associated with poorer treatment response to chemotherapy and worse prognosis in NSCLC patients treated with Platinum-based chemotherapy

\section{References}

1 Kosmidis P: Chemotherapy in NSCLC: historical review. Lung Cancer 2002;38:S19-22.

2 Hildebrandt MA, Gu J, Wu X: Pharmacogenomics of platinum-based chemotherapy in NSCLC. Expert Opin Drug Metab Toxicol 2009;5:745-755.

3 Gridelli C, Ferrara C, Del Gaizo F, Guerriero C, Nicolella D, Colantuoni G, Rossi A: Chemotherapy of advanced NSCLC in the elderly. Tumori 2002;88:S143-144.

- 4 Wu X, Ye Y, Rosell R, Amos CI, Stewart DJ, Hildebrandt MA, Roth JA, Minna JD, Gu J, Lin J, Buch SC, Nukui T, Ramirez Serrano JL, Taron M, Cassidy A, Lu C, Chang JY, Lippman SM, Hong WK, Spitz MR, Romkes M, Yang P: Genome-wide association study of survival in non-small cell lung cancer patients receiving platinumbased chemotherapy. J Natl Cancer Inst 2011;103:817-825.

-5 Wei SZ, Zhan P, Shi MQ, Shi Y, Qian Q Yu LK, Song Y: Predictive value of ERCC1 and XPD polymorphism in patients with advanced non-small cell lung cancer receiving platinum-based chemotherapy: a systematic review and meta-analysis. Med Oncol 2011;28:315-321.

-6 Hou XW, Son J, Wang Y, Ru YX, Lian Q, Majiti W, Amazouzi A, Zhou YL, Wang PX, Han ZC: Granulocyte colony-stimulating factor reduces cardiomyocyte apoptosis and improves cardiac function in adriamycininduced cardiomyopathy in rats. Cardiovasc Drugs Ther 2006;20:85-91.

7 Glaysher S, Yiannakis D, Gabriel FG, Johnson P, Polak ME, Knight LA, Goldthorpe Z, Peregrin K, Gyi M, Modi P, Rahamim J, Smith ME, Amer K, Addis B, Poole M, Narayanan A, Gulliford TJ, Andreotti PE, Cree IA: Resistance gene expression determines the in vitro chemosensitivity of non-small cell lung cancer (NSCLC). BMC Cancer 2009;9:300.

8 Li D, Zhou Q, Liu Y, Yang Y, Li Q: DNA repair gene polymorphism associated with sensitivity of lung cancer to therapy. Med Oncol 2012;29:1622-1628.

-9 Ehebauer M, Hayward P, Martinez-Arias A: Notch signaling pathway. Sci STKE 2006;2006:cm7.

10 Rose SL: Notch signaling pathway in ovarian cancer. Int J Gynecol Cancer 2009;19:564-566. 
Shi et al.: The Notch 3 Protein and Chemotherapy Response in Advanced NSCLC

11 Brzozowa M, Mielanczyk L, Michalski M, Malinowski L, Kowalczyk-Ziomek G, Helewski K, HarabinSlowinska M, Wojnicz R: Role of Notch signaling pathway in gastric cancer pathogenesis. Contemp Oncol (Pozn) 2013;17:1-5.

12 Li C, Zhang Y, Lu Y, Cui Z, Yu M, Zhang S, Xue X: Evidence of the cross talk between Wnt and Notch signaling pathways in non-small-cell lung cancer (NSCLC): Notch3-siRNA weakens the effect of LiCl on the cell cycle of NSCLC cell lines. J Cancer Res Clin Oncol 2011;137:771-778.

-13 Dang TP, Gazdar AF, Virmani AK, Sepetavec T, Hande KR, Minna JD, Roberts JR, Carbone DP: Chromosome 19 translocation, overexpression of Notch3, and human lung cancer. J Natl Cancer Inst 2000;92:1355-1357.

14 Haruki N, Kawaguchi KS, Eichenberger S, Massion PP, Olson S, Gonzalez A, Carbone DP, Dang TP: Dominantnegative Notch3 receptor inhibits mitogen-activated protein kinase pathway and the growth of human lung cancers. Cancer Res 2005;65:3555-3561.

15 Zheng Y, de la Cruz CC, Sayles LC, Alleyne-Chin C, Vaka D, Knaak TD, Bigos M, Xu Y, Hoang CD, Shrager JB, Fehling HJ, French D, Forrest W, Jiang Z, Carano RA, Barck KH, Jackson EL, Sweet-Cordero EA: A rare population of CD24(+)ITGB4(+)Notch(hi) cells drives tumor propagation in NSCLC and requires Notch3 for self-renewal. Cancer Cell 2013;24:59-74.

16 Park JT, Chen X, Trope CG, Davidson B, Shih Ie M, Wang TL: Notch3 overexpression is related to the recurrence of ovarian cancer and confers resistance to carboplatin. Am J Pathol 2010;177:1087-1094.

17 Menon S, Cox HC, Kuwahata M, Quinlan S, MacMillan JC, Haupt LM, Lea RA, Griffiths LR: Association of a Notch 3 gene polymorphism with migraine susceptibility. Cephalalgia 2011;31:264-270.

18 Wu F, Hu N, Li Y, Bian B, Xu G, Zheng Y: Galectin-3 genetic variants are associated with platinum-based chemotherapy response and prognosis in patients with NSCLC. Cell Oncol (Dordr) 2012;35:175-180.

19 Monteiro DR, Zuim PR, Pesqueira AA, Ribeiro Pdo P, Garcia AR: Relationship between anxiety and chronic orofacial pain of temporomandibular disorder in a group of university students. J Prosthodont Res 2011;55:154-158.

20 Roy B, Maksemous N, Smith RA, Menon S, Davies G, Griffiths LR: Two novel mutations and a previously unreported intronic polymorphism in the NOTCH3 gene. Mutat Res 2012;732:3-8.

21 Remmele W, Stegner HE: [Recommendation for uniform definition of an immunoreactive score (IRS) for immunohistochemical estrogen receptor detection (ER-ICA) in breast cancer tissue]. Pathologe 1987;8:138-140.

22 Dang L, Fan X, Chaudhry A, Wang M, Gaiano N, Eberhart CG: Notch3 signaling initiates choroid plexus tumor formation. Oncogene 2006;25:487-491.

23 Li L, Zeng H, Hou X, He X, Chen JX: Myocardial injection of apelin-overexpressing bone marrow cells improves cardiac repair via upregulation of Sirt3 after myocardial infarction. PLoS One 2013;8:e71041.

24 Zhang S, Loch AJ, Radtke F, Egan SE, Xu K: Jagged1 is the major regulator of Notch-dependent cell fate in proximal airways. Developmental dynamics : an official publication of the American Association of Anatomists 2013;242:678-686.

25 Ye YZ, Zhang ZH, Fan XY, Xu XL, Chen ML, Chang BW, Zhang YB: Notch3 overexpression associates with poor prognosis in human non-small-cell lung cancer. Med Oncol 2013;30:595.

26 Hou XW, Wang LF, Wang N, Pang D, Hui B, Zhou YL, He X: The G501C polymorphism of oxidized LDL receptor gene [OLR-1] is associated with susceptibility and serum C-reactive protein concentration in Chinese essential hypertensives. Clin Chim Acta 2008;388:200-203.

27 Lee SH, Jeong EG, Yoo NJ, Lee SH: Mutational analysis of NOTCH1, 2, 3 and 4 genes in common solid cancers and acute leukemias. APMIS 2007;115:1357-1363.

28 Maraver A, Fernandez-Marcos PJ, Herranz D, Canamero M, Munoz-Martin M, Gomez-Lopez G, Mulero F, Megias D, Sanchez-Carbayo M, Shen J, Sanchez-Cespedes M, Palomero T, Ferrando A, Serrano M: Therapeutic effect of gamma-secretase inhibition in KrasG12V-driven non-small cell lung carcinoma by derepression of DUSP1 and inhibition of ERK. Cancer Cell 2012;22:222-234.

29 Zhang S, Hou X, Zi S, Wang Y, Chen L, Kong B: Polymorphisms of receptor for advanced glycation end products and risk of epithelial ovarian cancer in Chinese patients. Cell Physiol Biochem 2013;31:525-531. 